\title{
Donor-acceptor conjugated systems: a neutral hydrophobic fluorescence probe
}

\begin{abstract}
Fluorescence probe with intramolecular charge transfer behavior has received a great deal of attention in recent years. Such molecule is highly sensitive to its local environment and plays critical role in many industrial and biological applications. Donor-acceptor conjugated systems based on diphenylpolyene are one of such example. The presence of conjugated chain length and the donor-acceptor substituent's at the appropriate position allows the molecule to cover a wide range of absorption and fluorescence wavelength and hence, the optical property of the molecule is attenuated easily. Thus, diphenylpolyenes are suitable candidate for various fluorescence probe applications. The above mentioned aspects have been discussed and summarized in this paper.
\end{abstract}

Keywords: absorption, fluorescence, fluorescence probe, charge transfer, diphenylpolyene
Volume 3 Issue 5 - 2016

\author{
Jagdeep Kumar, Naresh Kumar, Prasanta \\ Kumar Hota \\ Department of Chemistry, Hemvati Nandan Bahuguna \\ Garhwal University, India
}

Correspondence: Prasanta Kumar Hota, Department of Chemistry, School of Sciences, Srinagar, Garhwal ? 246176 Uttarakhand, India,Tel -251901,Email p.hota@hnbgu.ac.in

Received: October 20, 2016 | Published: December 05, 2016
Abbreviations: LE, locally excited; ICT, intramolecular charge transfer

\section{Introduction}

A fluorescence probe is a molecule that plays important role in sensing various biological ${ }^{1-5}$ and chemical processes. ${ }^{6-10}$ This includes cell imaging applications, ${ }^{1,2,5}$ studying the bio-molecular interaction (e.g. protein-lipid, protein-protein, small moleculeprotein interaction), ${ }^{6-10}$ characterization of microenvironment of organized assemblies, ${ }^{9,10}$ etc. The development of two photon absorbing fluorophore near-infrared wavelength region is one of the such example, that is used for cell imaging application because of its less photo-damaging activity towards live cells. ${ }^{5}$ Upon absorption of light, such fluorescence probe is excited to its higher energy level and returned back to ground state by emitting light at higher wavelength. This process is highly dependent upon the nature of surrounding environment and the structure of the molecule. A good fluorescence probe is characterized by its maximum absorption and emission wavelength, extinction coefficient, quantum yield of emission and large Stokes' shift value. ${ }^{11}$ A fluorescence probe is mainly classified into two categories: small organic molecules and biomolecule (e.g. protein, antibody and peptides). The protein such as Green fluorescent protein is based on fluorescent biomolecule that can anchored into a non-fluorescent biomolecule for probing the biological process. ${ }^{1,2}$ On the other hand, few extrinsic fluorescence probes based on small organic molecule were developed for tracking such biological process. ${ }^{3-10,12-24}$ Fluorescence probe can be attached to a biomolecule like protein, through amino or carboxyl or thiol groups and the biological events can be monitored using fluorescence techniques. The existing extrinsic fluorescence probes, however, have their own issues and limitation for such biological application. Some of the extrinsic probes have shorter absorption and emission wavelength, which makes them limited to use in medicinal application. Some of them have individual charge that rise the question of possible probe-biomolecular interaction and may interfere with the biological process. Some fluorophores are toxic, lesser solubility and therefore, not suitable for such purpose. Probing studies with fluorescence probe bearing neutral charge, greater sensitivity, higher absorption and fluorescence maximum are ideal, but are limited, e.g. prodan, diphenyl polyene, etc (Figure 1). Donor-acceptor based conjugated diphenyl polyene is one such fluorophore that play important role not only for the characterization of the structure, function of retinal-bound photoreceptors, ${ }^{25,26}$ but also act as a membrane probe. ${ }^{27}$ Extensive studies and review on the spectral properties of donor-acceptor conjugated systems are available in the literature ${ }^{28-35}$
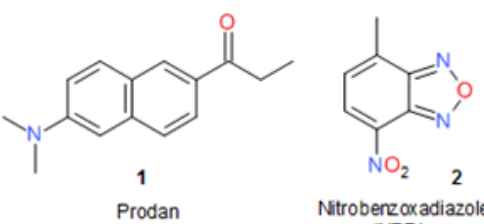
Nitroberzoxadiazole
(NBD)

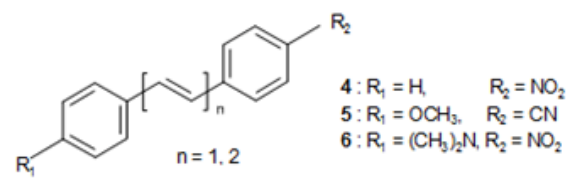

Figure I Structure of some of the neutral fluorescent probe that are used in tracking various chemical and biological processes.

\section{Diphenyl polyene as a fluorescence probe for future application}

In general, donor-acceptor substituted diphenyl ethenes and dienes show solvent dependent absorption and fluorescence properties. Some of the molecules exhibit dual fluorescence emission, which is highly dependent upon the local environment and substituent present on the molecule. ${ }^{36-41}$. For example, diarylethenes bearing donor group, methoxy and amine or acceptor group, cyano and nitro show fluorescence emission from their locally excited state (LE), whereas, in presence of both donor and acceptor substituents, cyano or nitro and methoxy and amine shows solvent polaritydependent dual fluorescence emission due to LE and intra molecular charge transfer (ICT) states. The synthesis of conjugated diphenyl polyenes are simple and easy through various condensation reaction including Wittig-Wadsworth-Emmons reaction. ${ }^{42}$ The presence of conjugated chain length and the donor-acceptor substituents at the appropriate position allows the molecule to cover a wide range of 
absorption and fluorescence wavelength as well as solubility. Hence, the optical properties of the molecule is attenuated easily. Some of these molecules with ICT phenomena are highly sensitive to their local environment and thus, diphenyl polyene systems are suitable for various fluorescence probe application.

Recently, several groups have shown that donor-acceptor based ethenes ${ }^{6-10}$ promised and serve as excellent neutral fluorescence probe for studying organized assemblies, membrane and protein systems. It is shown that the fluorescence intensity of some of these molecule is increased linearly with increasing protein concentration and act as a probe for protein quantification. ${ }^{7}$ Some of these donor-acceptor based diphenylpolyenes act as a probe for organized assemblies, ${ }^{9,10}$ lipid, vesicle and protein interaction study..$^{6-8,10,18,27}$ In general, these probes have restricted motion in the vesicles and fluoresce from LE state, whereas fluoresce from ICT state in micelles. Recently such type of donor-acceptor conjugated systems are also used as a sensor for detection of ions for various other applications. ${ }^{43-45}$

\section{Conclusion}

It is clear from the foregoing discussion that diphenyl polyene based fluorescent probes with charge transfer character is useful for several biological, industrial, and medical applications. In general, donor-acceptor diphenylpolyenes are neutral and better fluorescent at ambient temperature and relatively easy to prepare. Diphenylpolyenes substituted with appropriate donor-acceptor groups with charge transfer phenomena are highly sensitive to their local environment. Thus, development of novel fluorophore based on diphenylpolyene is definitely useful for probing various chemical and biological processes as discussed above.

\section{Acknowledgements}

PKH is gratefully acknowledged UGC, New Delhi India for providing UGC-FRPS start-up grant.

\section{Conflicts of interest}

Author declare there are no conflicts of interest.

\section{Funding}

None.

\section{References}

1. Tsien RY. The green fluorescent protein. Annu Rev Biochem. 1998;67:509-544.

2. Jakobs S, Andresen M, Wurm CA. Probes and tags to study biomolecular function. In: Miller LW (Eds.), Wiley-VCH Verlag $\mathrm{GmbH} \& \mathrm{Co}$. Weinheim, Germany. 2008. p. 73.

3. Kim HM, An MJ, Hong JH, et al. Two photon fluorescent probes for acidic vesicles in live cells and tissue. Angew Chem Int Ed Engl. 2008;47(12):2231-2234.

4. Goncalves MST. Fluorescent labeling of biomolecules with organic probes. Chem Rev. 2009;109(1):190-212.

5. Kobayashi H, Ogawa M, Alford R,et al. New strategies for fluorescent probe design in medical diagnostic imaging. Chem Rev 2010;110(5):2620-2640.

6. Lunardi $\mathrm{CN}$, Tedesco AC, Kurth TL, et al. The complex between 9-(n-decanyl)acridone and bovine serum albumin. Part 2. What do fluorescence probes probe? Photochem Photobiol Sci. 2003;2(9):954-959.
7. Singh AK, Hota PK. Ethenyl indoles as neutral hydrophobic fluorescence probes. J Phys Org Chem. 2007;20(8):624-629.

8. Shiraishi K, Sanji T, Tanaka M. A displacement assay for the sensing of protein interactions using sugar-tetraphenylethene conjugates. Tetrahedron Lett. 2010;51(48):6331-6333.

9. Palakollu V, Kanvah S. Diphenylpolyene-cholesterol conjugates as fluorescent probes for microheterogeneous media. J Photochem Photobiol A Chem. 2014;281:18-26.

10. Mazzoli A, Spalletti A, Carlotti B, et al. Spectroscopic investigation of interactions of new potential anticancer drugs with DNA and non-ionic micelles. J Phys Chem B. 2015;119(4):1483-1495.

11. Lakowicz JR. Principles of fluorescence spectroscopy. (3rd edn), Springer, USA. 2006.

12. Weber G, Farris FJ. Synthesis and spectral properties of a hydrophobic fluorescent probe: 6-Propionyl-2-(dimethylamino) naphthalene. Biochemistry. 1979;18(14):3075-3078.

13. Jones ME, Lentz BR. Phospholipid lateral organization in synthetic membranes as monitored by pyrene-labeled phospholipids: effects of temperature and prothrombin fragment 1 binding. Biochemistry. 1986;25(3):567-574.

14. Sohi JL, Splittgerber AG. The binding of coomassie brilliant blue to bovine serum albumin. A physical biochemistry experiment. $J$ Chem Edu. 1991;68(3):262-264.

15. Davis DM, Birch DJS. Extrinsic fluorescence probe study of human serum albumin using nile red. J Fluoresc. 1996;6(1):23-32.

16. Plasek J, Sigler K. Slow fluorescent indicators of membrane potential: a survey of different approaches to probe response analysis. JPhotochem Photobiol B Biol. 1996; 33(2):101-124.

17. Nakamaru Y, Sato C. Identical independent sites for dye ligand on bovine serum albumin demonstrated by multivariate analysis. Biochim Biophys Acta. 2000;1480(1-2):321-328.

18. Di Cesare N, Lakowicz JR. Wavelength-ratiometric probes for saccharides based on donor-acceptor diphenylpolyenes. J Photochem Photobiol A Chem. 2001;143(1):39-47.

19. Lapinski MM, Blanchard GJ. The role of phospholipid headgroups in mediating bilayer organization. Perturbations induced by the presence of a tethered chromophore. Chem Phys Lipids. 2007;150(1):12-21.

20. Li Q, Min J, Ahn YH, Namm J, et al. Styryl-based compounds as potential in vivo imaging agents for $\beta$-amyloid plaques. Chem Bio Chem. 2007;8(14):1679-1687.

21. Soh N, Makihara K, Ariyoshi T, et al. Phospholipid-linked coumarin: a fluorescent probe for sensing hydroxyl radicals in lipid membranes. Anal Sci. 2008;24(2):293-296.

22. Amdursky N, Huppert D. Auramine-O as a fluorescence marker for the detection of amyloid fibrils. J Phys Chem B. 2012;116(45):13389-13395.

23. Agazzi FM, Rodriguez J, Falcone RD, et al. Prodan dual emission feature to monitor BHDC interfacial properties changes with the external organic solvent composition. Langmuir . 2013;29(11):3556-3566.

24. Kisin FE, Shabat D. New repertoire of 'donor-two-acceptor NIR fluorogenic dyes. Bioorg Med Chem. 2013;21(12):3602-3608.

25. Dugave C, Demange L. Cis-Trans isomerisation of organic molecules and biomolecules: Implications and applications. Chem Rev. 2003;103(7):2475-2532.

26. Singh AK, Hota PK. Development of bacteriorhodopsin analogues and studies of charge separated excited states in the photoprocesses of linear polyenes. Photochem Photobiol. 2007;83(1):50-62. 
27. Lentz BR. Use of fluorescent probes to monitor molecula order and motions within liposome bilayers. Chem Phys Lipids. 1993;64(1-3):99-116.

28. Waldeck DH. Photoisomerisation dynamics of stilbenes. Chem Rev 1991;91(3):415-436.

29. Saltiel J, Sears DF, Ko DH, et al. Cis-trans-isomerisation of alkenes. In Horspool WHM \& Song PS (Eds.), CRC press, Boca Raton, New York. 1995. p.3-15.

30. Singh AK, Mahalaxmi GR. Photoprocesses of linearly conjugated $\mathrm{C}=\mathrm{C}$ polyenes. Proc Natl Acad Sci India Sect A. 2000;70:1-26.

31. Liu RSH, Hammond GS. Photochemical reactivity of polyenes: from dienes to rhodopsin, from microseconds to femtoseconds. Photochem Photobiol Sci. 2003;2: 835-844.

32. Gorner H, Kuhn HJ. Cis-trans photoisomerization of stilbenes and stilbene-like molecules. John Wiley \& Sons, Inc. 2007. p.1-117.

33. Sonoda Y, Shimoi Y, Goto M, et al. Fluorescence properties of (E,E,E)1,6-di(n-naphthyl)-1,3,5-hexatriene $(\mathrm{n}=1,2)$ : effects of internal rotation. J Phys Chem A. 2013;117(3):566-578.

34. Carlotti B, Kikas I, Skoric I, et al. Photophysics of push-pull distyrylfurans, thiophenes and pyridines by fast and ultrafast techniques. Chem Phys Chem. 2013;14(5):970-981.

35. Redwood CE, Kanvah S, Samudrala R, et al. Bicycle pedal photoisomerization of 1-phenyl-4-(4-pyridyl)-1,3-butadienes in glassy isopentane at 77 K. J Photochem Photobiol Sci. 2013;12:1754-1760.

36. Pines D, Pines E, Rettig W. Dual fluorescence and excited-state structural relaxations in donor-acceptor stilbenes. The J Phys Chem A 2003;107(2):236-242.
37. Gezawy HE, Rettig W, Lapouyade R. Model studies of spectral and photophysical characteristics of donor-acceptor-polyenes: dimethylamino-cyano-diphenylbutadiene. Chem Phys Lett 2005;401(1-3):140-148.

38. Rafiq S, Sen P. Dielectric controlled excited state relaxation pathways of a representative push-pull stilbene: a mechanistic study using femtosecond fluorescence up-conversion technique. J Chem Phys. 2013;138:084308

39. Hota PK, Singh AK. Donor-acceptor conjugated linear polyenes: A study of excited state intramolecular charge transfer, photoisomerization and fluorescence probe properties. J Fluoresc. 2014.

40. Kumar N, Kumar J, Hota PK. Donor-acceptor conjugated molecules: Charge transfer, mechanism and significance. Res Rev J Chem. 2015;4 87-91.

41. Agnihotri H, Vasu AK, Palakollu V, et al. Neutral and cationic pyridylbutadienes : solvatochromism and fluorescence response with sodium cholate. Photochem Photobiol Sci. 2015;14(12):2159-2167.

42. Wadsworth WS, Emmons WD. The utility of phosphonate carbanions in olefin synthesis. J Am Chem Soc. 1961;83(7):1733-1738.

43. Mashraqui SH, Betkar R, Ghorpade S, et al. A new internal charge transfer probe for the highly selective detection of $\mathrm{Zn}$ (II) by means of dual colorimetric and fluorescent turn-on responses. Sensors and Actuators B. 2012;174:299-305.

44. Shahid M, Misra A. A simple and sensitive intramolecular charge transfer fluorescent probe to detect $\mathrm{CN}$ - in aqueous media and living cells. Anal Methods. 2013;5:434-437.

45. Wang R, Ren $\mathrm{P}, \mathrm{Pu} \mathrm{S}$, et al. Novel sensitive sensors for $\mathrm{Cu} 2+$ and optical switching of photochromic dithienylethene derivatives. $J$ Photochem Photobiol A Chem. 2014; 294:44-53. 